Proceedings

\title{
Polymeric Magnetic Microparticles as Electrochemical Immunosensing Platforms ${ }^{\dagger}$
}

\author{
Celia Toyos-Rodríguez * Francisco Javier García-Alonso and Alfredo de la Escosura-Muñiz \\ NanoBioAnalysis Group-Department of Physical and Analytical Chemistry, University of Oviedo, Julián \\ Clavería 8, 33006 Oviedo, Spain; \\ * Correspondence: toyoscelia@uniovi.es; Tel.: +34-609577416 \\ + Presented at the 1st International Electronic Conference on Biosensors, 2-17 November 2020; Available \\ online: https://iecb2020.sciforum.net/.
}

Received: date; Accepted: date; Published: date

\begin{abstract}
Magnetic microparticles (MMPs) have been notably used as platforms in biosensing. Due to their magnetic behavior, they simplify purification and separation procedures, reducing time of analysis. They also allow sample preconcentration, minimizing matrix effects, what is of key relevance for applications using real samples. Even though there is a great number of commercially available MMPs, their performance is not always reliable. In this work we propose the synthesis of novel polymeric MMPs for their use as electrochemical immunosensing platforms. Initially, magnetic nanoparticles of a diameter of $12 \pm 2 \mathrm{~nm}$ and a saturation magnetization of $70 \mathrm{emu} / \mathrm{g}$ were synthesized and characterized. Then, they were encapsulated in a polymeric matrix of poly (lacticco-glycolic) acid (PLGA), generating MMPs with a diameter of $90 \pm 18 \mathrm{~nm}$. Later, MMPs were functionalized with polyethyleneimine (PEI) as an intermediate step for the immobilization of affinity proteins or antibodies, necessary in electrochemical immunosensing. This would allow the obtaining of MMPs comparable to the commercially available ones but possessing higher saturation magnetization. The use of such MMPs could facilitate the detection of analytes of interest in diagnostics, among other applications.
\end{abstract}

Keywords: magnetic nanoparticles; magnetic microparticles; polymers; immunosensors; electrochemistry

\section{Introduction}

Magnetic particles (MPs) have been widely used over the last years in analytical detection techniques such as bioimaging, drug delivery [1], wastewater treatment [2] or biosensing [3], due to their high binding capacity and magnetic properties [4]. In this broad range of applications, properties such as size or magnetic moment $(m)$ of MPs is crucial, as it is coercivity or remanent magnetization [5]. Regarding size, this parameter is of especial concern, especially in biomedicine in which a size lower than $100 \mathrm{~nm}$ is required to avoid phagocytosis and liver and spleen accumulation [6]. However, in biosensing larger MPs are preferred owing to an increased magnetization that facilitates separation of biomolecules.

Two main types of MPs are used for such applications: magnetic nanoparticles (MNPs), of a diameter from 5-100 nm, and magnetic microparticles (MMPs) of 1-5 $\mu \mathrm{m}$.

MNPs are mainly composed of iron oxides as magnetite $\left(\mathrm{Fe}_{3} \mathrm{O}_{4}\right)$ that present a low toxicity and stability under extreme conditions [7]. In addition, MNPs are generally synthesized in a diameter size lower than $20 \mathrm{~nm}$, permitting a superparamagnetic behavior, which is essential for assuring an appropriate tracking of the particles in the presence of an external magnetic field [8] while minimizing aggregation. 
However, size reduction entails a decrease in the $m$ of the MPs what affects their usefulness. To counter this point, MMPs have been proposed as an alternative, allowing the obtention of an increased $m$ due to the greater amount of magnetic material per particle [9].

Colloidally assembled MMPs have been proposed for maintaining superparamagnetic behavior and getting an increased $m$. These MMPs are characterized by consisting on the encapsulation of MNPs of high $m$ into polymeric matrices [10]. These MMPs can be obtained by different ways, ranging from polymer encapsulation [11] to emulsion-based bottom-up assembly [10].

In this work, MMPs have been obtained by polymer encapsulation, using magnetite nanoparticles and poly (D,L-lactide-co-glycolide) (PLGA) as encapsulation polymer. MNPs of magnetite with a high $m$ have been obtained by thermal-decomposition and used as magnetic seeds, while PLGA has been selected due to its biocompatibility and its facility for further functionalization, what it is essential for its use as biosensing platforms.

\section{Materials and Methods}

\subsection{Materials}

Benzyl ether $\left(\mathrm{C}_{14} \mathrm{H}_{14} \mathrm{O}, 98 \%\right)$, oleic acid $\left(\mathrm{C}_{18} \mathrm{H}_{34} \mathrm{O}_{2}, 99.9 \%\right)$ iron (III) acetylacetonate $\left(\mathrm{C}_{15} \mathrm{H}_{21} \mathrm{FeO}_{6}\right.$, 97\%), Resomer ${ }^{\circledast} \mathrm{RG} 503 \mathrm{H}$, Poly(D,L-lactide-co-glycolide) (PLGA) $\left(\left[\mathrm{C}_{3} \mathrm{H}_{4} \mathrm{O}_{2}\right] \times\left[\mathrm{C}_{2} \mathrm{H}_{2} \mathrm{O}_{2}\right] \mathrm{y}, 50: 50, \mathrm{Mw}\right.$ 24.000-38.000), Poly(vinyl alcohol) (PVA) ([- $\left.\mathrm{CH}_{2} \mathrm{CHOH}-\right]_{\mathrm{n}}, \mathrm{Mw} 40,000-70,000$ 87-90\% hydrolyzed) and Polyethyleneimine (PEI) $\left(\mathrm{H}\left(\mathrm{NHCH}_{2} \mathrm{CH}_{2}\right) \mathrm{nNH}_{2}, \mathrm{Mw} \sim 25,000\right)$ were purchased from SigmaAldrich. Petroleum ether $\left(40^{\circ}-60^{\circ}\right)$, 2-Propanol $\left(\mathrm{C}_{3} \mathrm{H}_{8} \mathrm{O}, 99.9 \%\right)$ and dichloromethane (DCM) $\left(\mathrm{CH}_{2} \mathrm{Cl}_{2}\right.$, $99.8 \%)$ were obtained from VWR. Oleylamine $\left(\mathrm{C}_{18} \mathrm{H}_{37} \mathrm{~N}, 80-90 \%\right)$ was supplied by Acros Organics.

\subsection{Synthesis and Characterization of MNPs}

MNPs were synthesized following a previously published procedure [12]. Briefly, $\mathrm{Fe}(\mathrm{acac}) 3$ was dispersed in a mixture of dibenzyl ether, oleylamine and oleic acid. The mixture was placed in a three-necked round-bottom flask and heated with vigorous mechanical stirring, refrigeration and inert atmosphere until reflux; then the heating was maintained for 15 min under the same conditions. Afterwards, heating was removed, and the reaction was maintained at vigorous stirring for $15 \mathrm{~min}$ more, to later reconnect the heating source and maintain the reaction under reflux for $2 \mathrm{~h}$. After that, heating was removed again, and the reaction was maintained under stirring for 30 min more until cooled down. The resultant particles were purified by washing with a mixture 1:1 in volume of 2propanol and light petroleum ether using an external magnetic disk and dried under vacuum. Dried particles were reconstituted in 2-propanol at a concentration of $\sim 100 \mathrm{mg} / \mathrm{mL}$.

The as-synthesized nanoparticles were characterized by transmission electron microscopy (TEM) using either a JEOL2000 EXII TEM, using a copper grid and an accelerating voltage of $160 \mathrm{kV}$. Size distribution was obtained by measuring the diameter of each particle with ImageJ. X-ray powder diffraction (XRPD) was also performed to obtain the composition profile and the crystallinity size of the particles, using CuK $\alpha 1,2$ radiation $(\lambda=1: 54056 \AA$ and $1.54439 \AA)$ in a Bragg-Brentano reflection configuration, on an PHILIPS $X^{\prime}$ PERT PRO Panalytical diffractometer in a $2 \theta$ range of $15-90^{\circ}$, with a step size of 0.03 . Composition of MNPs was analyzed by Fourier transformed infrared spectroscopy (FT-IR) using a FT-IR spectrometer Paragon 1000 from PerkinElmer (USA) using KBr disks.

Additionally, magnetic measurements were performed at room temperature using a PPMS-14T (Physical Property Measurement System).

\subsection{Synthesis and Characterization of Polymeric MMPs}

MMPs were synthesized following a polymer encapsulation procedure. First, a suspension of MNPs was added to a solution of PLGA and PVA $2 \%$ and sonicated during $1 \mathrm{~min}$. Next, the obtained MMPs were left in a rotary evaporator to facilitate encapsulation and hardening and afterwards they were washed three times with water using an external magnetic disk, and then resuspended in water. PLGA covered MMPs were also functionalized with a layer of PEI for facilitating further bioconjugations. First, PLGA covered MMPs were transferred to a $1.5 \mathrm{~mL}$ Eppendorf ${ }^{\circledR}$ and separated to 
the solution using a MagRack ${ }^{\circledR}$. Then, an aqueous solution of PEI was added and stirred using a Vortex during $1 \mathrm{~h}$. The MMPs@PEI were washed three-times with water and resuspended for furhter characterization. MMPs were characterized using a JEOL2000 EXII TEM, using a copper grid and an accelerating voltage of $160 \mathrm{kV}$. And by Dynamic Light Scattering using a Malvern Instruments Zetasizer Nano SZ with a solid-state He-Ne laser (wavelength $\lambda=633 \mathrm{~nm}$ ).

\section{Results}

\subsection{Sturctural Chracterization of MNPs}

MNPs can be synthesized following different procedures, going from co-precipitation, which allows the production of nanoparticles in an easiest way, to thermal- decomposition. In our case, this second method has been followed, using iron (III) acethylacetonate as precursor in the presence of oleic acid and oleylamine as surfactants (Figure 1A). In this process, controlling heating ramp is crucial in order to regulate the dimensions of the nanoparticles generated [13] and the polydispersity of the sample. In this case, temperature has been controlled indirectly, showing a linear increase until $220^{\circ} \mathrm{C}$ when temperature stabilizes and magnetic nucleus formation takes place [14].

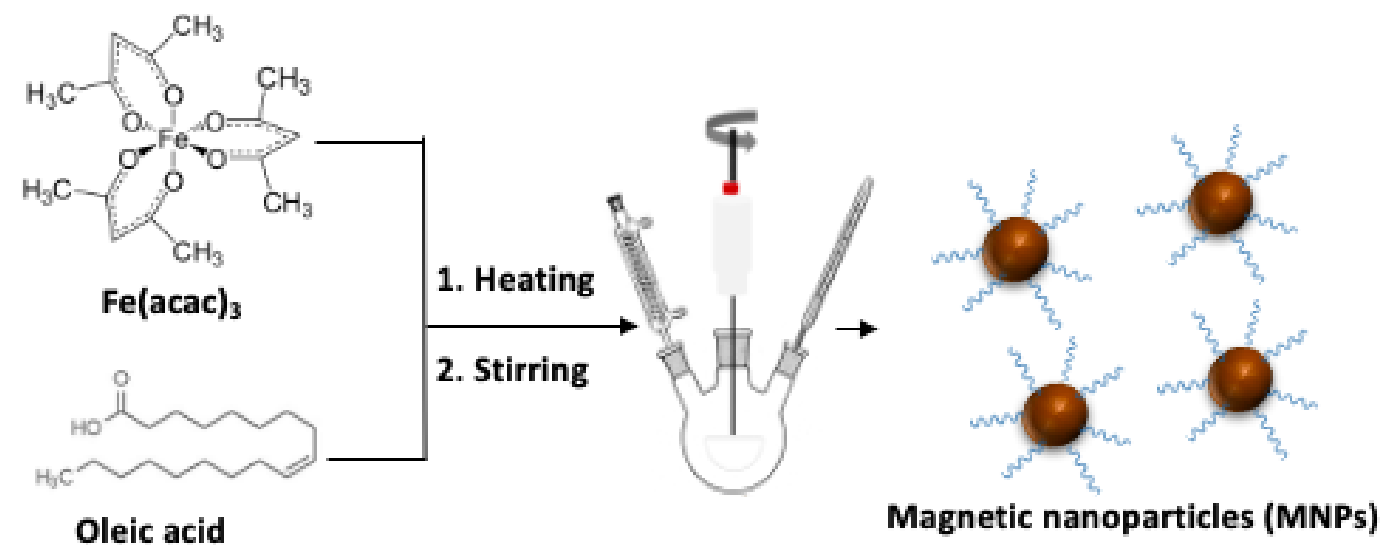

Figure 1. A. Schematic representation of the synthetic procedure for the formation of MNPs. B. Heating curve of the reaction among time, in which it is represented the burst nucleation phase and the magnetic nucleus formation phase.

The exhaustive control of temperature allows the formation of MNPs with an average diameter size of $15 \pm 5 \mathrm{~nm}$ according to TEM images (Figure 2), showing a reduced polydispersity of the sample, both in size and shape.

A

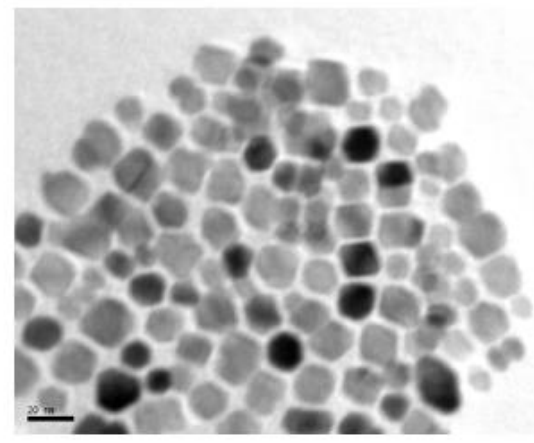

B

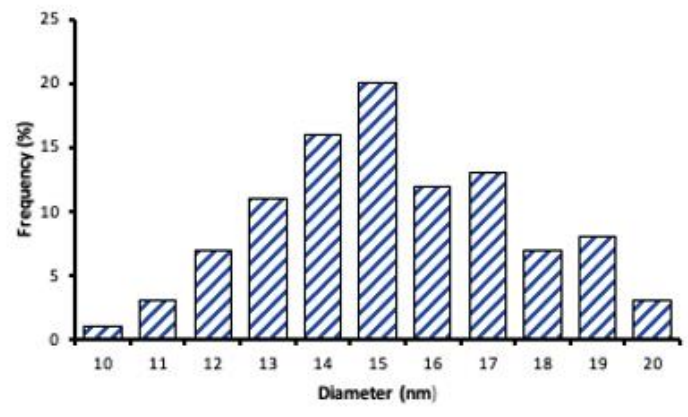

Figure 2. (A) TEM images of as-synthesized MNPs and (B) size distribution histogram. 
Diameter size has been compared with the estimated crystallinity size, in order to obtain the crystallinity grade of the sample. In this case, the estimation has been performed by X-Ray diffraction, using a Le Bail fitting method, revealing an apparent size of $12 \pm 2 \mathrm{~nm}$, what indicates that the nanoparticles are monocrystallines (Figure 3A). Moreover, the composition of MNPs has been studied using both X-Ray diffraction and IR spectroscopy. In the first case, characteristic diffraction peaks reveal that MNPs are composed of magnetite $\left(\mathrm{Fe}_{3} \mathrm{O}_{4}\right)$ (Figure 3B), what it is fundamental as magnetite easily oxidases into maghemite $\left(\gamma-\mathrm{Fe}_{2} \mathrm{O}_{3}\right)$, leading to a reduction in the magnetization of the nanoparticles [15].
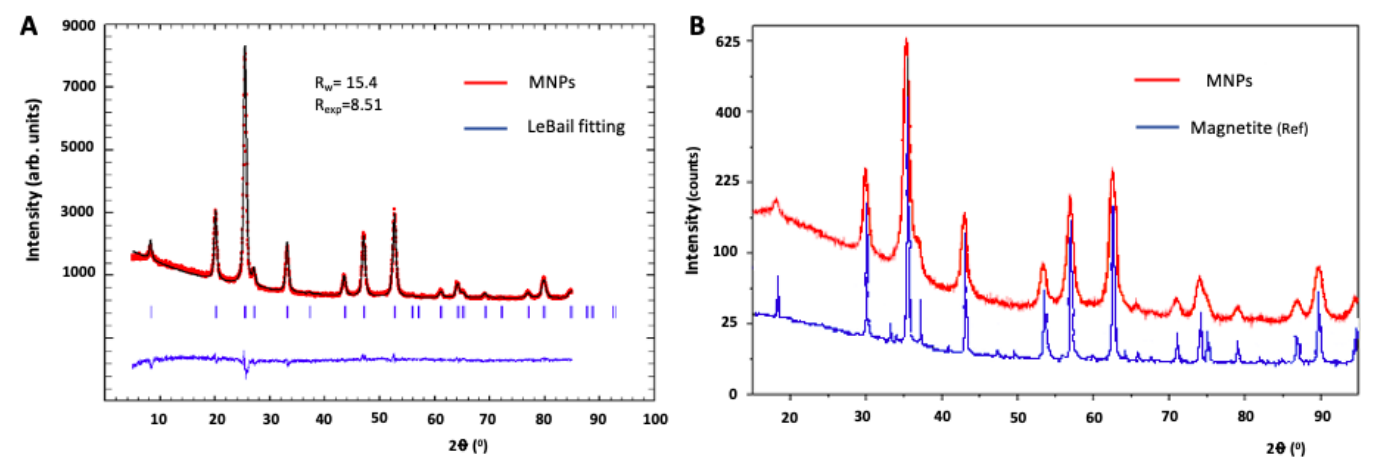

Figure 3. (A) LeBail fitting (blue line) and experimental data (red line) of MNPs. (B) X-Ray diffraction pattern of MNPs (red line) showing the correlation with pure magnetite (blue line) used as reference.

By IR, it has been also confirmed the presence of magnetite by the appearance of a band at 577 $\mathrm{cm}^{-1}$ due to magnetite solid-state vibrations. Additionally, the presence of bands at $2911 \mathrm{~cm}^{-1}, \mathrm{w}$, and $2842 \mathrm{~cm}^{-1}, \mathrm{w}$ indicate that MNPs are coated by oleates, as this bands correspond to the stretching of $\mathrm{CH}$ bonds. Bands at $1523 \mathrm{~cm}^{-1}, \mathrm{w}, \mathrm{br}$, and $1425 \mathrm{~cm}^{-1}, \mathrm{~m}$, br correspond to the antisymmetric and symmetric stretching of carboxylate groups of the oleate and $\mathrm{CH}_{2}$ deformation.

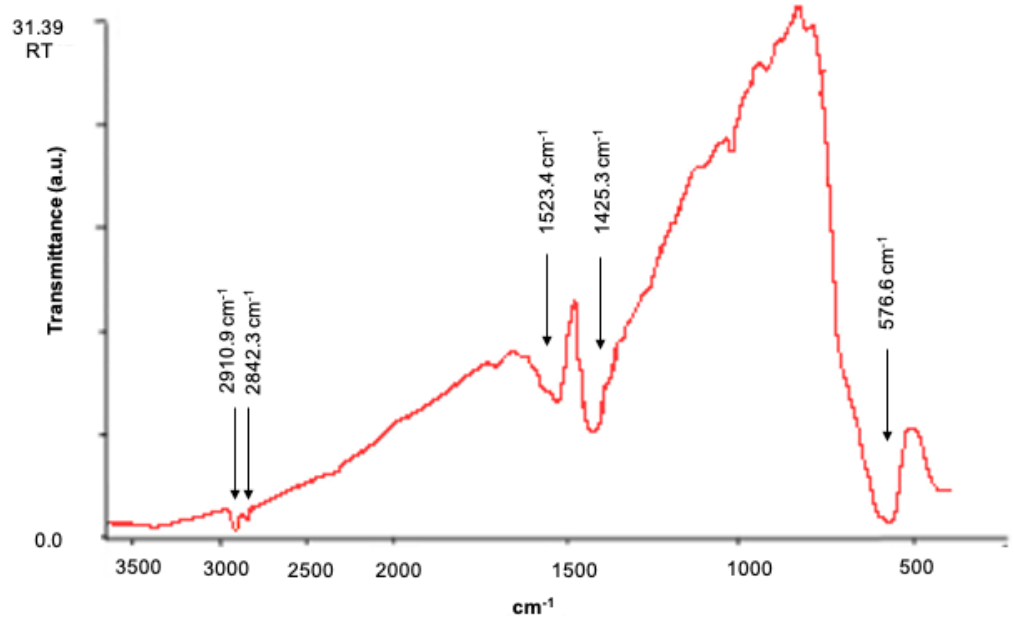

Figure 4. Infrared spectrum of MNPs showing the corresponding peaks of magnetite $\left(577 \mathrm{~cm}^{-1}\right)$ and of the oleate surrounding the NPs $\left(2910.9 \mathrm{~cm}^{-1}, 2842.3 \mathrm{~cm}^{-1}, 1523.4 \mathrm{~cm}^{-1}\right.$ and $\left.1425.3 \mathrm{~cm}^{-1}\right)$.

Once known the characteristics of the as-synthesized MNPs, the analysis of their magnetic behavior was performed. For that purpose, hysteresis curve of MNPs at room temperature was measured (Figure 5), showing a superparamagnetic behavior with a coercive field of 12 Oe and a saturation magnetization of $70 \mathrm{emu} / \mathrm{g}$, a value near the one for the bulk magnetite $(90 \mathrm{emu} / \mathrm{g})$ [16]. 
The above described results show that the MNPs synthesized in this work have ideal conditions both of polydispersity and magnetic behavior for its use as precursors for the formation of polymeric MMPs.

\subsection{Sturctural Chracterization of MMPs}

MMPs were synthesized by colloidally assemble of MNPs using a emulsion-based assembly method [11]. Briefly, a dispersion of MNPs in 2-propanol was mixed with PLGA solution and incorporated into a water phase of PVA. The contact of these two phases facilitates the assemble of polymeric MMPs. In this preliminary work, the as-synthesized MMPs were subsequent functionalized with PEI to facilitate its further functionalization with affinity proteins as NAV or antibodies that allow its use as immunosensing platforms. The obtained MMPs@PEI were characterized by measuring the magnetic behavior at room temperature. The hysteresis curve reveals that the Ms of the MMPs@PEI is lower than the obtained for the MNPs as the Ms is a parameter proportional to the weight of the sample and decreases among functionalization [17]. However, the assemble of MNPs into a cluster assures the formation of a higher magnetic moment $(\mathrm{m})$ compared to the obtained for the MNPs.

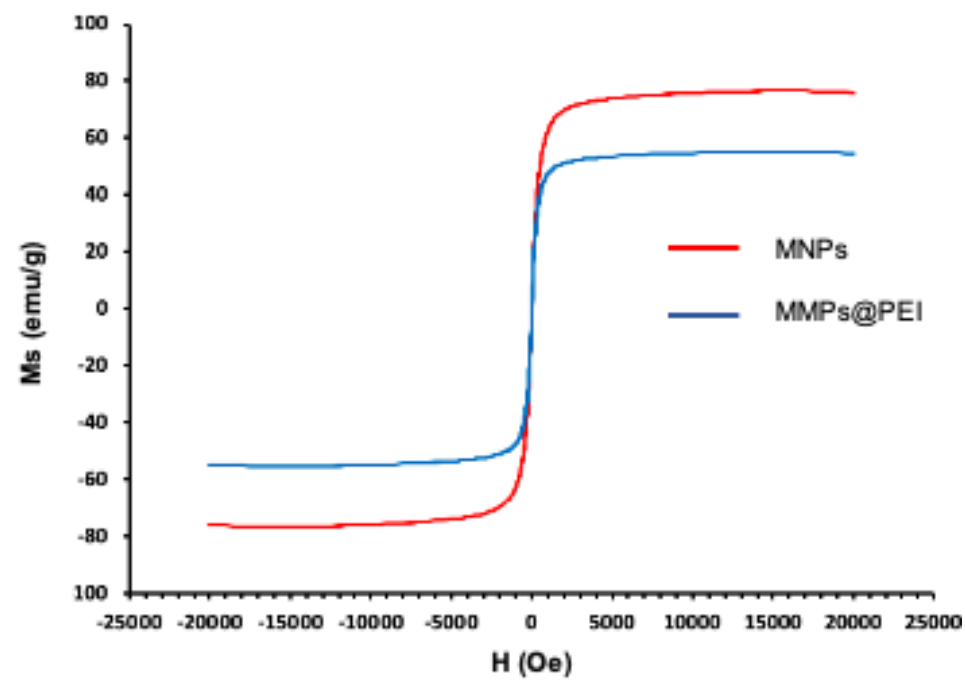

Figure 5. Hysteresis curve of MNPs (red line) and PEI functionalized MMPs (blue line).

MMPs have been characterized also in terms of size and shape of the particles generated. TEM measurements have been performed, showing that the as-synthesized MMPs have a size-distribution of $90 \pm 18 \mathrm{~nm}$, with a spherical shape and an appropriate polydispersity (Figure 6).
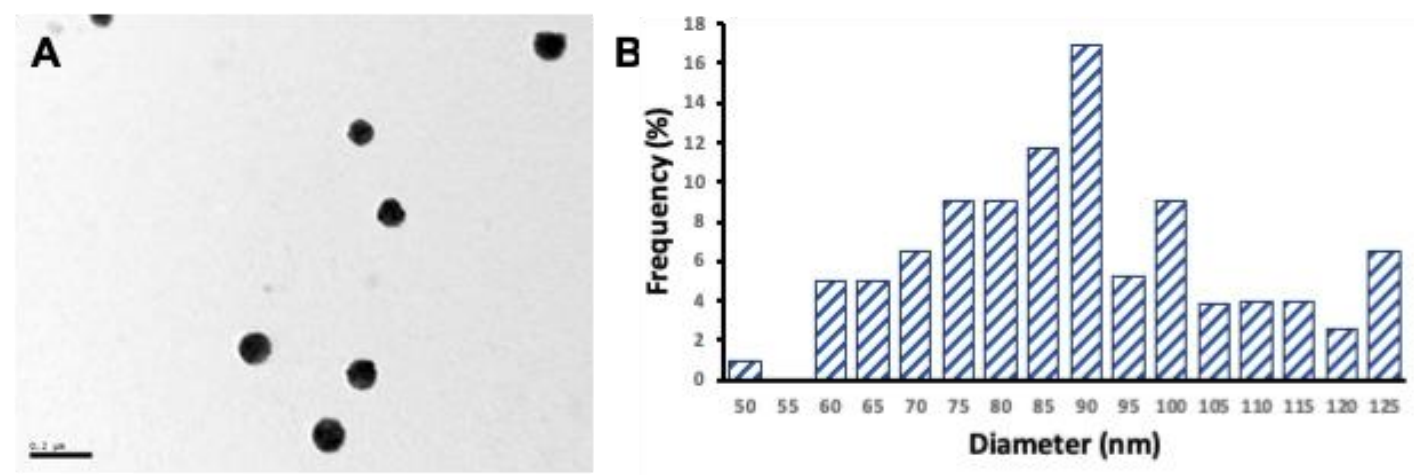

Figure 6. (A) TEM micrographs of PEI functionalized MMPs and (B) size distribution histogram of the previously mentioned particles. 
In order to determine if the functionalization has occurred appropriately, it has been recorded by dynamic light scattering the $\zeta$-potential of MMPs after the functionalization with PLGA and PEI, detecting a notable change from the neutral surface charge of MMPs@PLGA $(0.645 \mathrm{mV})$ to the positive surface charge of PEI functionalized MMPs $(15.6 \mathrm{mV})$ due to the presence of amine groups (Figure 7).

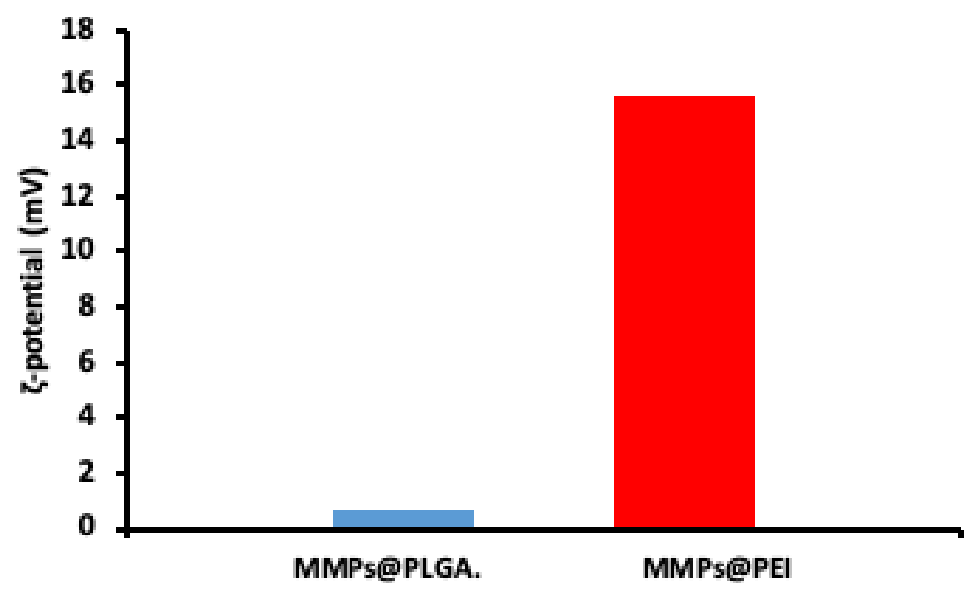

Figure 7. $\zeta$-potential of MMPs@PLGA (blue) and MMPs@PEI (red) showing the difference in the surface charge among functionalization.

\section{Discussion}

Firstly, the use of a thermal-decomposition method has allowed to obtain MNPs with an appropriate size and polydispersity while maintaining an elevated $\mathrm{Ms}$ of $70 \mathrm{emu} / \mathrm{g}$. Moreover, the structural characterization of the as-synthesized nanoparticles showed that they are mainly composed of magnetite with few presences of maghemite or other iron oxide forms, what explains the high Ms obtained.

Concerning the formation of PLGA-MMPs, in this work MMPs of $90 \pm 18 \mathrm{~nm}$ with favorable polydispersity and stability in aqueous media have been obtained. Additionally, these particles have been functionalized with PEI in order to facilitate its ulterior bioconjugation with affinity proteins as NAV or antibodies, what it is essential for their use as immunosensing platform. Magnetic behavior of MMPs@PEI shows a reduction in the Ms of the particle due to the increase of non-magnetic material.

All the above-mentioned information notes that MMPs synthesized in this work could be suitable for its use as immunosensing platforms, even though further research is required to investigate the bioconjugation of these MMPs with affinity molecules.

Author Contributions: C.T.-R.; conceived, designed and performed the experiments, data analyzes, writingreview and editing; F.J.G.-A. conceptualization, supervision, writing-review and editing; A.d.l.E.-M.; supervision, writing-review and editing and funding acquisition. All authors have read and agreed to the published version of the manuscript.

Funding: This research was funded by the FC-GRUPIN-ID/2018/000166 project from the Asturias Regional Government, the CTQ2017-86994-R project from the Spanish Ministry Economy and Competitiveness (MINECO) and supported by the IUTA/Gijón Council under the grant SV-20-GIJON-10. C. Toyos-Rodríguez thanks the MICINN (Spain) for the award of a FPI Grant (PRE2018-084853). A. de la Escosura-Muñiz acknowledges the MICINN (Spain) for the "Ramón y Cajal" Research Fellow (RyC-2016-20299).

Conflicts of Interest: The authors declare no conflict of interest.

\section{References}

1. Reddy, L.H.; Arias, J.L.; Nicolas, J.; Couvreur, P. Magnetic Nanoparticles: Design and Characterization, Toxicity and Biocompatibility, Pharmaceutical and Biomedical Applications. Chem. Rev. 2012, 112, 58185878 . 
2. Tang, S.C.N.; Lo, I.M.C. Magnetic nanoparticles: Essential factors for sustainable environmental applications. Water Res. 2013, 47, 2613-2632.

3. Xu, Y.; Wang, E. Electrochemical biosensors based on magnetic micro/nano particles. Electrochim. Acta 2012, $84,62-73$.

4. Chen, Y.-T.; Kolhatkar, A.G.; Zenasni, O.; Xu, S.; Lee, T.R. Biosensing Using Magnetic Particle Detection Techniques. Sensors 2017, 17, 2300.

5. Graham, D.L.; Ferreira, H.A.; Freitas, P.P. Magnetoresistive-based biosensors and biochips. Trends Biotechnol. 2004, 22, 455-462.

6. Moghimi, S.M. Mechanisms of splenic clearance of blood cells and particles: towards development of new splenotropic agents. Adv. Drug Deliv. Rev. 1995, 17, 103-115.

7. Nasir Baig, R.B.; Nadagouda, M.N.; Varma, R.S. Magnetically retrievable catalysts for asymmetric synthesis. Coord. Chem. Rev. 2015, 287, 137-156.

8. Netto, C.G.C.M.; Toma, H.E.; Andrade, L.H. Superparamagnetic nanoparticles as versatile carriers and supporting materials for enzymes. J. Mol. Catal. B Enzym. 2013, 85-86, 71-92.

9. Zhang, Y.; Xu, J.; Li, Q.; Cao, D.; Li, S. The effect of the particle size and magnetic moment of the $\mathrm{Fe}_{3} \mathrm{O}_{4}$ superparamagnetic beads on the sensitivity of biodetection. AIP Adv. 2019, 9, 015215.

10. Bai, F.; Wang, D.; Huo, Z.; Chen, W.; Liu, L.; Liang, X.; Chen, C.; Wang, X.; Peng, Q.; Li, Y. A Versatile Bottom-up Assembly Approach to Colloidal Spheres from Nanocrystals. Angew. Chem. Int. Ed. 2007, 46, 6650-6653.

11. Bigall, N.C.; Wilhelm, C.; Beoutis, M.-L.; García-Hernandez, M.; Khan, A.A.; Giannini, C.; Sánchez-Ferrer, A.; Mezzenga, R.; Materia, M.E.; Garcia, M.A.; et al. Colloidal Ordered Assemblies in a Polymer Shell -A Novel Type of Magnetic Nanobeads for Theranostic Applications. Chem. Mater. 2013, 25, 1055-1062.

12. Toyos-Rodríguez, C.; Calleja-García, J.; Torres-Sánchez, L.; López, A.; Abu-Dief, A.M.; Costa, A.; Elbaile, L.; Crespo, R.D.; Garitaonandia, J.S.; Lastra, E.; et al. A Simple and Reliable Synthesis of Superparamagnetic Magnetite Nanoparticles by Thermal Decomposition of Fe(acac)3. J. Nanomater. 2019, 2019, doi:10.1155/2019/2464010.

13. Guardia, P.; Pérez-Juste, J.; Labarta, A.; Batlle, X.; Liz-Marzán, L.M. Heating rate influence on the synthesis of iron oxide nanoparticles: the case of decanoic acid. Chem. Commun. 2010, 46, 6108.

14. Kemp, S.J.; Ferguson, R.M.; Khandhar, A.P.; Krishnan, K.M. Monodisperse magnetite nanoparticles with nearly ideal saturation magnetization. RSC Adv. 2016, 6, 77452-77464.

15. Santoyo Salazar, J.; Perez, L.; de Abril, O.; Truong Phuoc, L.; Ihiawakrim, D.; Vazquez, M.; Greneche, J.-M.; Begin-Colin, S.; Pourroy, G. Magnetic Iron Oxide Nanoparticles in 10-40 nm Range: Composition in Terms of Magnetite/Maghemite Ratio and Effect on the Magnetic Properties. Chem. Mater. 2011, 23, 1379-1386.

16. Tao, K.; Dou, H.; Sun, K. Interfacial coprecipitation to prepare magnetite nanoparticles: Concentration and temperature dependence. Colloids Surfaces A Physicochem. Eng. Asp. 2008, 320, 115-122.

17. Wu, W.; He, Q.; Jiang, C. Magnetic Iron Oxide Nanoparticles: Synthesis and Surface Functionalization Strategies. Nanoscale Res. Lett. 2008, 3, 397-415.

Publisher's Note: MDPI stays neutral with regard to jurisdictional claims in published maps and institutional affiliations.

2020 by the authors. Submitted for possible open access publication under the terms and conditions of the Creative Commons Attribution (CC BY) license (http://creativecommons.org/licenses/by/4.0/). 